\title{
Structured Clinical Case Presentation for Assessment of Undergraduates during Initial Clinical Postings
}

\author{
Vandana Jain $^{1}$, Rajni Sharma ${ }^{1}$
}

\begin{abstract}
Structured Clinical Case Presentation (SCCP) was designed as a modification of the long case to more objectively and systematically assess the communication, examination and analytical skills of undergraduate students. Students' perception of the assessment by the long case or SCCP was noted using questionnaires and feedback collected from the examining faculty regarding the strengths and limitations of the new method.
\end{abstract}

Key words: assessment, case presentation, objectivity, structured

\section{Introduction}

The long case presentation is the conventional method to assess undergraduate medical students at the end of their clinical postings and during their final examinations. Although this is a good tool to assess students' ability to synthesize clinical findings, power of reasoning and in-depth theoretical knowledge, it lacks structure, objectivity and consistency. It often assesses the end-product of history and examination, rather than the process itself (Sood, 2001). For students in the early phase of their clinical training, assessment of their communication skills with the patient and family, and the method of examination, is useful and important. Undergraduate medical students have their first short posting in Pediatrics during the $4^{\text {th }}$ semester. The objectives of this posting are to acquaint the students with eliciting history and performing physical examination, particularly anthropometry, in children; counseling of parents regarding nutrition, immunization etc and management of common illnesses of childhood.

${ }^{1}$ Department of Paediatrics

All India Institute of Medical Sciences, New Delhi, India

Corresponding Author

$D r^{1}$ Vandana Jain, $M D$

Fellow of GSMC-FAIMER Regional Institute,

Associate Professor, Department of Pediatrics,

Adjunct Faculty,

Center for Medical Education and Technology,

All India Institute of Medical Sciences,

New Delhi, India.

Email : drvandanajain@gmail.com
The assessment at the end of this posting comprises of a theory examination (4 short answer questions, 45 minutes, and viva for 5-10 minutes) and a long case. For the long case, each student is allotted one child patient in the pediatric ward for a detailed history, examination and synthesis of findings. The time allotted for this is 45 minutes, at the end of which the examiner evaluates the student, spending approximately 20-30 minutes with each student. Since evaluation of the long case is unstructured, the discussion is focused more on the differential diagnosis of the patient or on one particular aspect of the history, and does not provide a comprehensive assessment of the students' history-taking and examination skills. Thus, there is a mismatch between the objectives of the posting and the assessment. Also, there is marked variability in the difficulty level of the cases as well as the scoring pattern of examiners, which makes this method of assessment highly subjective. We designed a Structured Clinical Case Presentation (SCCP) as a modification of the conventional long case presentation (LCP) to be conducted with the objective of comparing the students' perception of assessment by long case and SCCP and assessing the strengths and limitations of SCCP.

\section{Methodology}

This prospective study was conducted at the Department of Pediatrics, All India Institute of Medical Sciences, New Delhi from June 2007 to May 2008. Approval for the study was given by the Institute's Dean. The study participants 
were one batch of undergraduate medical students $(n=43)$ and the faculty members involved in their teaching and assessment $(n=6)$.

Students were posted to the Pediatric Outpatient Department in 4 groups, of 10-12 students during their $4^{\text {th }}$ semester. The first and third groups of students $(n=22)$ were evaluated by the long case method and the second and fourth groups $(n=21)$ were assessed by the SCCP format.

The method of assessment for each group of students was explained to them at the beginning of their posting. For the SCCP groups, checklists for the different tasks were also provided at the beginning of their posting. The faculty members were also requested to align their teaching with the assessment method.

\section{Structured clinical case presentation (SCCP):}

Students were allotted cases of similar difficulty level by drawing lots. Each student had to perform two history and two examination tasks on the patient, displayed on a bedside card. Task 1 was to take the relevant history, task 2 was to take one particular aspect of history in detail (e.g., birth, immunization, developmental, nutritional etc.) and answer a related question/counsel parents, task 3 was to perform one anthropometric examination (height, weight, length, head circumference or mid arm circumference) and task 4 was to perform a general physical examination/ examination of one system. Table 1 shows a sample bedside card.

Table 1: Sample bedside task card

Task 1: Take the relevant history and present summary and analysis of the case to the examiner.

Task 2: Take nutritional history and present to the examiner. Counsel the mother regarding appropriate weaning foods for the infant (to be observed by the examiner).

Task 3: Measure the length of the infant (to be observed by the examiner).

Task 4: Perform examination of the cardio-vascular system (to be observed by the examiner).

Students were given 20 minutes for the workup, after which the examiner joined them. For tasks 1 and 2, the history was presented to the examiner. The second part of task 2 and both examination tasks were performed in front of the examiner. The students were evaluated according to previously prepared guidelines for the history tasks (1 and 2) and checklists for the examination tasks ( 3 and 4 ). Table 2shows the guidelines for evaluating task 1 and Table 3 shows a sample checklist for the examination task 3 . The checklist also awarded points to the establishment of a rapport and attention to details while conducting a physical examination. Feedback on students' performance (based on the guidelines/ checklist) was provided to the students immediately after the assessment.

Table 2: Guidelines for evaluation of Task 1

\begin{tabular}{lc}
\hline Point & $\begin{array}{c}\text { Maximum Marks } \\
(\mathbf{1 0 )}\end{array}$ \\
\hline Basic patient information & 2 \\
Chief complaints with duration & 4 \\
Course of illness & 4 \\
Relevant positive and negative history & 4 \\
Discussion of probable and differential diagnosis & 4 \\
Interaction with the informant and child & 2 \\
\hline
\end{tabular}


Table 3: Sample checklist for evaluation of Task 3 (Height Measurement)

\begin{tabular}{lc}
\hline Point & $\begin{array}{c}\text { Maximum Marks } \\
\text { (10) }\end{array}$ \\
\hline Procedure explained to child and parent & 1 \\
Footwear removed & 1 \\
Child made to stand with feet together & 1 \\
Heels, buttocks and occiput touch the wall/ vertical board & 2 \\
Head in Frankfurt plane & 2 \\
Horizontal headboard/ hard surface brought down to touch the head & 1
\end{tabular}

\section{Collection of Feedback:}

A questionnaire based on a 5-point Likert scale (1: strongly disagree to 5: strongly agree) was used for taking feedback (SCCP and LCP groups). This questionnaire was validated before using by discussing it with colleagues from the Medical Education Unit and a few students. It comprised of 16 questions grouped into 4 areas: acquisition of required knowledge and skills, teaching by the faculty, adequacy of the assessment in testing communication and examination skills and objectivity and fairness of assessment. The mean response scores in these areas were compared between the long case and SCCP groups by Student's t-test. The examiners' feedback was also taken regarding the strengths and limitations of SCCP.

\section{Results}

The marks scored by the students in both the groups were similar. The long case and SCCP groups scored $4.8 \pm 0.6$ and $4.9 \pm 0.7$, respectively, out of $7 . \overline{5}(\mathrm{p}=0.6)$ in the practical examination, and $4.2 \pm 0.3$ and $4.4 \pm 0.6$, respectively, out of 7.5 , in the combined theory and viva examination.

The mean (SD) of the feedback response scores of the two groups is compared in Table 1.

A statistically significant higher score was assigned by the SCCP group to the adequacy of assessment in testing communication and examination skills. The difference in scores in objectivity and fairness also approached statistical significance.
The feedback from the examiners $(n=6)$ was as follows:

\section{Strengths:}

1. In the SCCP group, the students had clearer goals for learning and were more motivated to learn proper techniques of history and examination.

2. The teaching was more focused in terms of imparting these skills.

3. This method was more effective in assessing psychomotor domain and equally effective as conventional long case for cognitive domain.

4. It was more objective and ensured greater uniformity in evaluation.

5. It provided immediate feedback and improved student learning.

\section{Limitations}

1. Preparing and validating checklists required a lot of ground work.

2. The time needed for the assessment of each student was longer.

\section{Discussion}

We have attempted to create some innovations in the conventional long case to modify it to the SCCP. Our aim was to retain the advantages of long case (assessing the holistic approach to a patient and ability to interpret the history to reach a provisional diagnosis) but at the same time surmount its limitations (not directly observing the process, lack of structure, bias and subjectivity) and make it a more valid and reliable tool for assessment of undergraduates in the early part of their clinical training. 
The SCCP offers the advantages of a structured format with checklists/ guidelines for evaluation. The history and examination tasks together provide a good assessment of cognitive, psychomotor and affective domains. In addition, the checklists provided to the students at the beginning of their posting serve as learning tools.

The students as well as faculty work towards the common goal of learning the correct methods of eliciting all aspects of history, examination and counseling of parents. With the conventional assessment tools that focus more on cognitive domains, the emphasis on learning the correct examination techniques and establishment of physical signs is low. It is a common observation that medical graduates are found lacking in these basic psychomotor skills that should have been well-established during undergraduate training (Chan-Yan et al, 1988, Gleeson, 1992). The provision of immediate specific feedback to the students based on the checklists also improves their learning. At the same time, if many students perform poorly on a given task or miss a particular step, it serves as a feedback to the teachers that the topic needs to be taught in a better way. Objective Structured Clinical Examination (OSCE) and Objective Structured Long Examination Record (OSLER) are two assessment tools that have also been developed to improve objectivity (Harden \& Gleeson 1979, Gleeson, 1997). However, OSCE has drawbacks such as compartmentalization of the case preventing assessment of the holistic approach to the patient, practical difficulties in setting up several stations and use of exhaustive checklists which are difficult to prepare and validate.

The OSLER method uses a 10-item analytical record: four items on history, three items on examination and one item each on appropriate investigations, management and clinical acumen. This method is more suitable for final year undergraduate medical students and post graduate students. In conclusion, the SCCP method seems to have a good scope for use as an assessment tool for students in the early phase of their clinical training.

\section{References}

Sood, R., (2001). Long Case Examination - can it be Improved? Journal, Indian Academy of Clinical Medicine, 2, pp. 251-255.

Chan-Yan, C., Gillies, J.H., Ruedy, J. Montaner, J.S.G, \& Marshal, S.A., 1988. Clinical Skills of Medical Residents: a Review of Physical Examination, Canadian Medical Association Journal, 139, 7, pp. 629-632.

Gleeson, F., 1992. Defects in Postgraduate clinical skills as Revealed by Objective Structured Long Examination Record (OSLER), Irish Medical Journal, 85, 1, pp. 11-14.

Harden, R.M. \& Gleeson, F.A., (1979). Assessment of Clinical Competence using an Objective Structured Clinical Examination (OSCE), Medical Education, 13, 1, pp. 41-54.

Gleeson, F. (1997). AMEE Medical Education guide No 9: Assessment of Clinical Competence using the Objective Structured Long Examination Record (OSLER), Medical Teacher, 19, pp.7-14. 